\title{
Numerical analysis of the radio-frequency single-electron transistor operation
}

\author{
Valentin O. Turin and Alexander N. Korotkov \\ Department of Electrical Engineering, University of California, Riverside, CA 92521-0204.
}

(October 31, 2018)

We have analyzed numerically the response and noiselimited charge sensitivity of a radio-frequency single-electron transistor (RF-SET) in a non-superconducting state using the orthodox theory. In particular, we have studied the performance dependence on the quality factor $Q$ of the tank circuit for $Q$ both below and above the value corresponding to the impedance matching between the coaxial cable and SET.

\section{INTRODUCTION}

An important drawback of the conventional singleelectron transistor ${ }^{1,2}$ (SET) is its relatively large output resistance which should be much larger than the quantum resistance $R_{Q}=h / 4 e^{2} \simeq 6.5 \mathrm{k} \Omega$. This limits the operation frequency of the prospective integrated single-electron circuits $^{3,4}$ and imposes a severe frequency limitation for individual SETs used nowadays as electrometers. Estimating the total capacitance of a wire delivering SET signal from a cryostat to outside electronics as $1 \mathrm{nF}$, we get the time constant on the order of $1 \mathrm{nF} \times 10^{5} \Omega=10^{-4} \mathrm{~s}$; therefore the operating frequency is limited to few $\mathrm{kHz}$, which is a typical value achievable by conventional SET setups. ${ }^{5}$ While the operating frequency can be significantly increased by placing a preamplifier in a close vicinity of the SET,, 7 the preferred at present solution of the problem is the use of the radio-frequency $\mathrm{SET}^{8}$ (RF-SET) which in many instances has already replaced the traditional SET setup.

The principle of the RF-SET operation is somewhat similar to the operation of the radio-frequency superconducting quantum interference device ${ }^{9}$ (RF-SQUID) and is based on the microwave reflection ${ }^{8,10^{-15}, 17,16,18,19}$ from a tank (LC) circuit containing the SET (Fig. 1); another possibility is to use the transmitted wave. ${ }^{20,21}$ The measured SET input signal changes effective SET resistance and affects the intensity and the phase of the reflected (or transmitted) wave that is later sensed by either homodyne detection or simple rectification (to separate incoming and reflected waves a directional coupler can be used). The high operation frequency of the RF-SET is due to the signal propagation by the microwave, so that the SET does not need to charge the whole output wire, while the tank circuit tuned in resonance works as an impedance transformer providing a better match between the effective SET differential resistance $R_{d}\left(\sim 10^{5} \Omega\right)$ and the microwave cable wave impedance $R_{0}$ (typically $50 \Omega$ ).

For a good matching the "unloaded" quality factor $Q \equiv \sqrt{\left(L_{T} / C_{T}\right)} / R_{0}$ of the tank circuit consisting of inductance $L_{T}$ and capacitance $C_{T}$ should be comparable to $\sqrt{R_{d} / R_{0}} \sim 50$. While a much lower value, $Q=6$, was used in the first experiment, ${ }^{8}$ the values close to the matching condition (sometimes even higher) are typically used at present. Increase of the $Q$-factor obviously decreases the RF-SET bandwidth limited by $\sim \omega / 2 Q_{L}$ where $\omega \approx 1 / \sqrt{L_{T} C_{T}}$ is the "carrier" microwave frequency and $Q_{L}$ is the "loaded" $Q$-factor, which also takes into account the effect of the SET (see below). The straightforward design $^{8}$ shown in Fig. 1 can be somewhat modified to reduce the bandwidth further for a dense multiplexing; ${ }^{14}$ however, in this paper we will consider only the original design.

The RF-SET bandwidth as wide as $100 \mathrm{MHz}$ has been demonstrated $^{8}$ using a relatively high carrier frequency $\omega / 2 \pi=1.7 \mathrm{GHz}$ and relatively low $Q$-factor $Q=6$. However, to improve experimental RF-SET sensitivity, it happens to be advantageous to reduce the carrier frequency by few times (to reduce the noise contribution from the amplifier) and also increase $Q$ (closer to the impedance matching regime), so that a practical bandwidth at present is about $10 \mathrm{MHz}$ (for example, the bandwidth of $7 \mathrm{MHz}$ for the carrier frequency of $332 \mathrm{MHz}$ has been reported in Ref. ${ }^{12}$ ).

The high operation frequency of the RF-SET makes it easily possible to avoid the $1 / f$ noise limitation of the SET sensitivity which is typically dominant at frequencies $f \lesssim 10^{4} \mathrm{~Hz}$, and so work in the region of the shot noise limited sensitivity. ${ }^{22,23}$ Though experimentally the contribution from the amplifier noise is still comparable or larger than the SET noise, a relatively rapid improvement of the RF-SET charge sensitivity from $1.2 \times 10^{-5} e / \sqrt{\mathrm{Hz}}$ at $1.1 \mathrm{MHz}$ in the first experiment $^{8}$ to the value $3.2 \times 10^{-6} e / \sqrt{\mathrm{Hz}}(4.8 \hbar$ in energy units) at $2 \mathrm{MHz}$ reported in Ref. ${ }^{12}$ assures that the pure shot noise limit will be achieved pretty soon. (The above numbers can be compared with the sensitivity $2 \times 10^{-5} \mathrm{e} / \sqrt{\mathrm{Hz}}$ at $4.4 \mathrm{kHz}$ of a purely conventional SET reported in Ref. $^{5}$, the value $8 \times 10^{-6} e / \sqrt{\mathrm{Hz}}$ at $10 \mathrm{~Hz}$ for the "stacked" SET, ${ }^{24}$ and the sensitivity $6 \times 10^{-6} e / \sqrt{\mathrm{Hz}}$ at $45 \mathrm{~Hz}$ for the SET made of carbon nanotubes. $^{25}$ )

One of important potential applications of the RFSET is for the readout of the charge qubits in a solidstate quantum computer. ${ }^{11,16-19,26}$ The possibility of a single-shot qubit measurement requires fast enough distinguishing between two charge states to avoid significant qubit evolution during measurement. This requires sufficiently wide RF-SET bandwidth and most importantly good enough charge sensitivity. The estimates ${ }^{11}$ show that the single-shot qubit measurement is almost within 
the reach of present-day RF-SET performance; however, reliable measurement still requires significant improvement in sensitivity. This makes very important the question of ultimate (theoretical) RF-SET sensitivity. The ultimate RF-SET sensitivity is also a crucial parameter for monitoring quantum dynamics of nanomechanical resonators ${ }^{27-29}$ and for a variety of RF-SET applications as an electrometer in classical single-electron devices.

In spite of significant experimental activity on RFSETs, we are aware of only few theoretical papers on the RF-SETs. The basic theory of the shot noise limited charge sensitivity of the RF-SET has been developed in Ref. ${ }^{30}$. A similar theory has been applied to the sensitivity analysis for the RF-SET-based micromechanical displacement detector. ${ }^{31-33}$ Some theoretical analysis of the transmission-type RF-SET can be found in Ref. ${ }^{21}$. The theory of a somewhat related device, radio-frequency Bloch-transistor, has been developed in Ref. ${ }^{34}$. In our opinion, the RF-SET definitely requires further theoretical attention, since many questions about RF-SET performance has not yet been answered theoretically.

In this paper we extend the theory of Ref. ${ }^{30}$ to the case of arbitrary $Q$-factor of the tank circuit, removing the assumption (strongly violated in the present-day experiments) of $Q$ being much smaller than the impedance matching value. We calculate the response and sensitivity of the normal-metal RF-SET and optimize these magnitudes numerically over the rf wave amplitude and the SET background charge. Then we study the dependence of the optimized RF-SET response and sensitivity on the tank $Q$-factor, operation temperature, SET resistance, carrier frequency, and SET asymmetry due to asymmetric biasing. Some results of this paper have been presented earlier in a short form. ${ }^{35}$

\section{MODEL AND CALCULATION METHODS}

The schematic of the RF-SET used in our analysis is shown in Fig. 1. The SET consists of two tunnel junctions with capacitances $C_{1 j}$ and $C_{2 j}$ and resistances $R_{1}$ and $R_{2}$. The SET is coupled via gate capacitance $C_{g}$ to the measured charge source (for example, a single-electron box or a similar structure), which is characterized by the charge $q_{S}$ and capacitances $C_{S 1}$ and $C_{S 2}$ to the SET leads (the total source capacitance is $C_{S}=C_{S 1}+C_{S 2}$ ). Assuming constant $q_{S}$ (neglecting backaction from the SET), it is easy to show that the SET coupled to the charge source is equivalent to the simple double-junction SET structure with parameters

$$
\begin{aligned}
& C_{1}=C_{1 j}+\frac{C_{g} C_{S 1}}{C_{g}+C_{S}}, \\
& C_{2}=C_{2 j}+\frac{C_{g} C_{S 2}}{C_{g}+C_{S}}, \\
& q_{0}=q_{00}+q_{S} \frac{C_{g}}{C_{g}+C_{S}},
\end{aligned}
$$

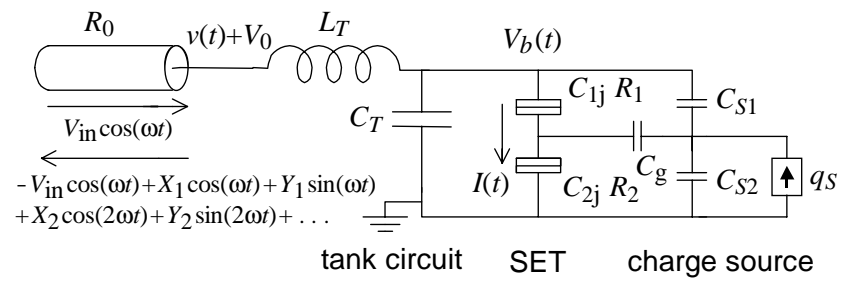

FIG. 1. Schematic of the RF-SET. The current $I(t)$ through the SET (two tunnel junctions with capacitances $C_{1 j}$ and $\left.C_{2 j}\right)$ affects the quality factor of the tank circuit $\left(L_{T}\right.$ and $C_{T}$ ) and therefore the amplitude and phase of the reflected $\mathrm{rf}$ wave propagating along coaxial cable $\left(R_{0}\right)$. The change of the source charge $q_{S}$ changes the effective SET background charge $q_{0}$ and can be monitored via measurement of the reflected $\mathrm{rf}$ wave.

where $C_{1}$ and $C_{2}$ are effective junction capacitances (the total SET island capacitance is $C_{\Sigma}=C_{1}+C_{2}$ ), and the total induced charge of the SET island $q_{0}$ is the sum of the initial background charge $q_{00}$ and the contribution from the measured charge source. For numerical results we have used the "orthodox" model ${ }^{1}$ for a normal-metal SET (see Appendix).

\section{Linear analysis of the reflected wave}

The current $I(t)$ through the SET affects the quality factor of the tank circuit consisting of inductance $L_{T}$ and capacitance $C_{T}$, while the contribution to the tank circuit capacitance is neglected, assuming $C_{T} \gg$ $\max \left[C_{1 j}, C_{2 j}, C_{S 1} C_{S 2} /\left(C_{S 1}+C_{S 2}\right)\right]$. (This condition is usually well satisfied experimentally; it also allows us to neglect the effect of single electron jumps on the tank circuit oscillations.) The quality factor of the tank circuit is also affected by the wave impedance $R_{0}$ of the cable. For a simple linear analysis let us substitute the SET by an effective differential resistance $R_{d}$. Then, assuming the case of weak damping, we can simply add the contributions from $R_{0}$ and $R_{d}$, so that the total ("loaded") quality factor of the tank circuit is

$$
\begin{aligned}
& Q_{L}=\left(1 / Q+1 / Q_{S E T}\right)^{-1}, \\
& Q=\frac{\sqrt{L_{T} / C_{T}}}{R_{0}}, Q_{S E T}=\frac{R_{d}}{\sqrt{L_{T} / C_{T}}},
\end{aligned}
$$

where the "unloaded" quality factor $Q$ corresponds to absence of the SET, $R_{d}=\infty$, while $Q_{S E T}$ corresponds to the damping by the SET only, $R_{0}=0$. Notice that $Q$ is fixed by the RF-SET design, while $Q_{S E T}$ and therefore $Q_{L}$ depend on the operating conditions; so even though $Q_{L}$ is a more physically meaningful quantity than $Q$, in this paper we consider unloaded $Q$ as an independent parameter and call it as $Q$-factor.

For the incoming voltage wave $\hat{V}_{i n} \exp (\mathrm{i} \omega t)$, the reflected wave $\alpha \hat{V}_{i n} \exp (\mathrm{i} \omega t)$ is determined by the complex reflection coefficient $\alpha$ : 


$$
\alpha=\frac{Z-R_{0}}{Z+R_{0}}, \quad Z=\mathrm{i} \omega L_{T}+\frac{1}{\mathrm{i} \omega C_{T}+1 / R_{d}} .
$$

Since the measured charge signal changes the SET resistance $R_{d}$, the RF-SET response is proportional to $d\left(\alpha \hat{V}_{i n}\right) / d R_{d}$. Using the first order approximation close to the resonant frequency $\omega_{0}=1 / \sqrt{L_{T} C_{T}}$ of the tank circuit:

$$
Z \approx \frac{L_{T} / C_{T}}{R_{d}}+2 \mathrm{i} \sqrt{L_{T} / C_{T}} \frac{\Delta \omega}{\omega_{0}}, \Delta \omega=\omega-\omega_{0}
$$

(in particular, this approximation neglects the shift of the resonant frequency, which is a second-order effect) it is easy to get

$$
\frac{d \alpha}{d R_{d}} \approx \frac{-2 R_{0}}{R_{d}^{2}} \frac{Q^{2}}{\left(1+\frac{Q^{2}}{R_{d} / R_{0}}\right)^{2}} \frac{1}{\left(1+2 \mathrm{i} Q_{L} \Delta \omega / \omega_{0}\right)^{2}} .
$$

For fixed values of $R_{0}$ and $R_{d}$, this expression increases with $Q$ for small $Q$, thus showing the need of $Q$-factor increase to achieve a better RF-SET response. The maximum is reached at

$$
Q=\sqrt{R_{d} / R_{0}},
$$

which is the case of practically matched impedances, $Z \approx R_{0}$, at frequencies close to resonance, and also corresponds to the condition $Q=Q_{S E T}=2 Q_{L}$. Notice that in this case the reflection practically vanishes, $\alpha \approx 0$.

Actually, Eq. (8) is not really relevant, because the rf amplitude $V_{\text {in }}$ should depend on $Q$ in order to maintain approximately constant amplitude $\left|\hat{V}_{b}\right|$ of the SET bias voltage oscillations, which is determined by the voltage scale of the Coulomb blockade. Taking into account the relation

$$
\hat{V}_{i n} / \hat{V}_{b}=\left(Z+R_{0}\right)\left(\mathrm{i} \omega C_{T}+1 / R_{d}\right) / 2,
$$

we obtain

$$
\frac{d \alpha}{d R_{d}}\left|\frac{\hat{V}_{i n}}{\hat{V}_{b}}\right| \approx \frac{-R_{0}}{R_{d}^{2}} \frac{Q}{1+\frac{Q^{2}}{R_{d} / R_{0}}} \frac{\left|1+2 \mathrm{i} Q_{L} \Delta \omega / \omega_{0}\right|}{\left(1+2 \mathrm{i} Q_{L} \Delta \omega / \omega_{0}\right)^{2}},
$$

which is somewhat similar to Eq. (8) and also reaches maximum at $Q=\sqrt{R_{d} / R_{0}}$. Notice that this condition optimizes only the RF-SET response, while the shotnoise-limited sensitivity can (and as will be seen later does) have completely different dependence on $Q$.

\section{Full analysis of the reflected wave}

The linear analysis discussed above can be used only as an estimate because of the significant nonlinearity of the SET current-voltage $(I-V)$ dependence. For a more exact analysis ${ }^{30}$ we use the Kirchhoff's rules taking into account the current $I(t)$ through the SET. Let us separate the voltage $V_{0}+v(t)$ at the end of the rf cable into the dc component $V_{0}$ (which can be supplied via the bias-tee $\left.{ }^{8}\right)$ and $\mathrm{rf}$ component $v(t)=V_{\text {in }}(t)+V_{\text {out }}(t)$, where $V_{i n}(t)=V_{\text {in }} \cos \omega t$ is the incoming wave (from now on we do not use complex representation) and $V_{\text {out }}(t)$ is the outgoing reflected wave. The differential equation for the rf component $v(t)$ is

$$
\begin{aligned}
\ddot{v} / \omega_{0}^{2}+\dot{v} / Q \omega_{0}+v= & 2\left(1-\omega^{2} / \omega_{0}^{2}\right) V_{\text {in }} \cos \omega t \\
& -R_{0}[I(t)-\langle I\rangle],
\end{aligned}
$$

where $\langle I\rangle$ is the current through the SET averaged over time much longer than $\omega^{-1}$, and the time dependence of the SET current $I(t)$ can be found self-consistently from the time dependence of its bias voltage

$$
V_{b}(t)=V_{0}+v(t)+\left[2 V_{i n} \omega \sin \omega t+\dot{v}(t)\right] Q / \omega_{0} .
$$

In this paper we assume that the rf frequency is small compared to the frequency of electron tunneling through the SET, $\omega \ll I / e$, so that the SET shot noise is a small contribution compared to the deterministic part of the SET current $I(t)$, which is calculated using de $I-V$ curve and $V_{b}(t)$ (actually, it is still OK if this condition is not satisfied during some fraction of the period due to Coulomb blockade, since the small current does not affect the oscillations significantly).

In a steady state (assuming that the induced SET charge $q_{0}$ does not change with time) the reflected wave can only contain the incoming frequency $\omega$ and its overtones:

$$
V_{\text {out }}(t)=-V_{\text {in }} \cos \omega t+\sum_{n=1}^{\infty}\left[X_{n} \cos n \omega t+Y_{n} \sin n \omega t\right] .
$$

We separate the term $-V_{\text {in }} \cos \omega t$ mainly to follow the notations of Ref. ${ }^{30}$, even though it really makes sense only for a low- $Q$ case at $\omega \approx \omega_{0}$, when all $X_{n}$ and $Y_{n}$ are small. Using the substitution $v(t)=\sum_{n=1}^{\infty}\left[X_{n} \cos n \omega t+\right.$ $\left.Y_{n} \sin n \omega t\right]$ in Eq. (12), we find the coefficients $X_{n}$ and $Y_{n}$ as

$$
\begin{aligned}
X_{n}= & R_{0} Q \frac{n \tilde{\omega} a_{n}-Q\left(1-n^{2} \tilde{\omega}^{2}\right) b_{n}}{n^{2} \tilde{\omega}^{2}+Q^{2}\left(1-n^{2} \tilde{\omega}^{2}\right)^{2}} \\
& +\frac{2 Q^{2}\left(1-\tilde{\omega}^{2}\right)^{2}}{\tilde{\omega}^{2}+Q^{2}\left(1-\tilde{\omega}^{2}\right)^{2}} V_{i n} \delta_{1 n}, \\
Y_{n}= & -R_{0} Q \frac{n \tilde{\omega} b_{n}+Q\left(1-n^{2} \tilde{\omega}^{2}\right) a_{n}}{n^{2} \tilde{\omega}^{2}+Q^{2}\left(1-n^{2} \tilde{\omega}^{2}\right)^{2}} \\
& +\frac{2 Q \tilde{\omega}\left(1-\tilde{\omega}^{2}\right)}{\tilde{\omega}^{2}+Q^{2}\left(1-\tilde{\omega}^{2}\right)^{2}} V_{i n} \delta_{1 n},
\end{aligned}
$$

where

$$
a_{n}=2\langle I(t) \sin n \omega t\rangle, \quad b_{n}=2\langle I(t) \cos n \omega t\rangle
$$

(averaging is over the oscillation period), $\tilde{\omega} \equiv \omega / \omega_{0}$ is the normalized frequency, $\delta_{1 n}$ is the Kronecker symbol, 
and the current $I(t)$ is calculated self-consistently using the SET bias voltage

$$
\begin{aligned}
V_{b}(t)= & V_{0}+2 Q \frac{\omega}{\omega_{0}} V_{i n} \sin \omega t+\sum_{n=1}^{\infty}\left[\left(X_{n}+\frac{Q n \omega}{\omega_{0}} Y_{n}\right)\right. \\
& \left.\times \cos n \omega t+\left(Y_{n}-\frac{Q n \omega}{\omega_{0}} X_{n}\right) \sin n \omega t\right] .
\end{aligned}
$$

Because of the resonant behavior of the tank circuit at $Q \gg 1$, the contribution of overtones $(n \geq 2)$ in the reflected signal is always small if $\omega \approx \omega_{0}$ (since the overtones are far from resonance). This can be easily seen from Eqs. (15)-(16), especially in the case $\omega=\omega_{0}$, when they are significantly simplified:

$$
\begin{aligned}
& X_{n}=R_{0} Q \frac{n a_{n}-Q\left(1-n^{2}\right) b_{n}}{n^{2}+Q^{2}\left(1-n^{2}\right)^{2}}, \\
& Y_{n}=-R_{0} Q \frac{n b_{n}+Q\left(1-n^{2}\right) a_{n}}{n^{2}+Q^{2}\left(1-n^{2}\right)^{2}} .
\end{aligned}
$$

Therefore, a linear (one-frequency) approximation in which only $X_{1}$ and $Y_{1}$ are taken into account, works very well in this case. In our numerical analysis we used the result of the linear approximation as a starting point of the iterative procedure $\left[V_{b}(t) \rightarrow\left(a_{n}, b_{n}\right) \rightarrow\left(X_{n}, Y_{n}\right) \rightarrow\right.$ $V_{b}(t) \rightarrow \ldots$ to solve selfconsistently Eqs. (15)-(18) taking into account few (typically $3-5$ ) overtones. We have checked numerically that the account of overtones typically gives a small correction in the case of a reasonably large $Q$-factor and $\omega \approx \omega_{0}$.

In the linear approximation the SET bias voltage has only one frequency component: $V_{b}(t)=V_{0}+A_{b} \sin (\omega t+$ $\phi)$ and therefore for the calculation of $a_{1}$ and $b_{1}$ [see Eq. (17)] the SET can be simply replaced by the effective resistance

$$
R_{d}=\frac{\pi A_{b}}{\int_{0}^{2 \pi} I\left(V_{0}+A_{b} \sin x\right) \sin x d x},
$$

where $I(V)$ is the SET current-voltage dependence. [Notice that $\int_{0}^{2 \pi} I\left(V_{0}+A_{b} \sin x\right) \cos x d x=0$, i.e. there is no effective reactance contribution.]

Hence, this linear (one-frequency) approximation is completely equivalent to the case of a resistor instead of the SET, considered in the previous subsection. The only new condition is a selfconsistent relation between the effective resistance $R_{d}$ and the amplitude $A_{b}$ of the SET bias voltage. The amplitude $A_{b}$ (which depends on $R_{d}$ ) can be calculated either using Eq. (10) in which $A_{b}=\left|\hat{V}_{b}\right|$ or using Eq. (18), which gives

$$
A_{b}=\sqrt{\left[Q \tilde{\omega}\left(2 V_{i n}-X_{1}\right)+Y_{1}\right]^{2}+\left(X_{1}+Q \tilde{\omega} Y_{1}\right)^{2}},
$$

while the components $X_{1}$ and $Y_{1}$ are given by equations

$$
X_{1}=\frac{2 Q^{2}}{Q^{2}+\tilde{R}} \frac{\tilde{\omega}^{2}+\left(1-\tilde{\omega}^{2}\right) \tilde{R} \frac{1+\left(1-\tilde{\omega}^{2}\right) \tilde{R}}{Q^{2}+\tilde{R}}}{\tilde{\omega}^{2}+\frac{Q^{2}\left[1+\left(1-\tilde{\omega}^{2}\right) \tilde{R}\right]^{2}}{\left(Q^{2}+\tilde{R}\right)^{2}}} V_{i n},
$$

$$
Y_{1}=\frac{2 \tilde{\omega} Q}{Q^{2}+\tilde{R}} \frac{-\frac{Q^{2}\left[1+\left(1-\tilde{\omega}^{2}\right) \tilde{R}\right]}{Q^{2}+\tilde{R}}+\left(1-\tilde{\omega}^{2}\right) \tilde{R}}{\tilde{\omega}^{2}+\frac{Q^{2}\left[1+\left(1-\tilde{\omega}^{2}\right) \tilde{R}\right]^{2}}{\left(Q^{2}+\tilde{R}\right)^{2}}} V_{i n},
$$

where $\tilde{R} \equiv R_{d} / R_{0} \gg 1$ (these equations can obviously be rewritten in a shorter way; however, they become less transparent to analyze).

The equations (23)-(24) significantly simplify in the case $\omega=\omega_{0}$ :

$$
\begin{aligned}
& X_{1}=\frac{2 Q^{2}\left(Q^{2}+\tilde{R}\right)}{\left(Q^{2}+\tilde{R}\right)^{2}+Q^{2}} V_{i n}, \\
& Y_{1}=\frac{-2 Q^{3}}{\left(Q^{2}+\tilde{R}\right)^{2}+Q^{2}} V_{i n},
\end{aligned}
$$

from which it is clear that $\left|Y_{1} / X_{1}\right| \ll 1$ for $Q \gg 1$, and therefore

$$
V_{b}(t) \approx V_{0}+\frac{2 Q \tilde{R} V_{i n}}{Q^{2}+\tilde{R}} \sin \omega t=V_{0}+2 Q_{L} V_{i n} \sin \omega t .
$$

\section{Response and noise-limited sensitivity}

So far we have implicitly assumed that the SET current $I(t)$ depends on time only because of the periodic time dependence of the SET bias voltage $V_{b}(t)$. However, $I(t)$ has also a small noise component, the magnitude of which depends on the bias voltage and therefore also has a periodic time dependence. The shot noise of the SET current leads to the fluctuations of the parameters $a_{n}$ and $b_{n}$ defined by Eq. (17) and consequently to the fluctuations of the reflected wave quadratures $X_{n}$ and $Y_{n}$. Since the noise of $X_{n}$ and $Y_{n}$ can be meaningfully discussed only at frequencies less than $\omega / Q_{L}$, which is much less than the typical frequency $I / e$ of electron tunneling in the SET, it is sufficient to consider the low-frequency limit of the SET shot noise.

The low-frequency spectral densities of $a_{n}$ and $b_{n}$, and their mutual spectral density can be calculated as

$$
\begin{aligned}
& S_{a n}=4\left\langle S_{I}(t) \sin ^{2} n \omega t\right\rangle, \quad S_{b n}=4\left\langle S_{I}(t) \cos ^{2} n \omega t\right\rangle, \\
& S_{a n, b n}=2\left\langle S_{I}(t) \sin 2 n \omega t\right\rangle,
\end{aligned}
$$

where the averaging is over the oscillation period, $S_{I}(t)$ is the low- (zero-) frequency spectral density of the SET current (see Appendix), and the time dependence comes from oscillating bias voltage $V_{b}(t)$. Consequently, the low-frequency spectral densities of $X_{n}$ and $Y_{n}$ fluctuations, and their mutual spectral density are

$$
\begin{aligned}
S_{X n}= & c_{n}^{2}\left\langle S_{I}(t) \sin ^{2} n \omega t\right\rangle+d_{n}^{2}\left\langle S_{I}(t) \cos ^{2} n \omega t\right\rangle \\
& -c_{n} d_{n}\left\langle S_{I}(t) \sin 2 n \omega t\right\rangle, \\
S_{Y n}= & d_{n}^{2}\left\langle S_{I}(t) \sin ^{2} n \omega t\right\rangle+c_{n}^{2}\left\langle S_{I}(t) \cos ^{2} n \omega t\right\rangle \\
& +c_{n} d_{n}\left\langle S_{I}(t) \sin 2 n \omega t\right\rangle, \\
S_{X n, Y n} & =c_{n} d_{n}\left\langle S_{I}(t) \cos 2 n \omega t\right\rangle \\
& +\frac{1}{2}\left(d_{n}^{2}-c_{n}^{2}\right)\left\langle S_{I}(t) \sin 2 n \omega t\right\rangle,
\end{aligned}
$$


where

$$
\begin{aligned}
c_{n} & =\frac{2 R_{0} Q n \tilde{\omega}}{n^{2} \tilde{\omega}^{2}+Q^{2}\left(1-n^{2} \tilde{\omega}^{2}\right)^{2}}, \\
d_{n} & =\frac{2 R_{0} Q^{2}\left(1-n^{2} \tilde{\omega}^{2}\right)}{n^{2} \tilde{\omega}^{2}+Q^{2}\left(1-n^{2} \tilde{\omega}^{2}\right)^{2}} .
\end{aligned}
$$

Notice much simpler equations for $S_{X_{1}}$ and $S_{Y_{1}}$ in the case $\omega=\omega_{0}$ :

$$
\begin{aligned}
& S_{X_{1}}=4 R_{0}^{2} Q^{2}\left\langle S_{I}(t) \sin ^{2} \omega t\right\rangle, \\
& S_{Y_{1}}=4 R_{0}^{2} Q^{2}\left\langle S_{I}(t) \cos ^{2} \omega t\right\rangle .
\end{aligned}
$$

The change of the measured charge $q_{S}$ can in principle be monitored via the change of any quadrature $X_{n}$ or $Y_{n}$. The corresponding responses can be defined as the derivatives $d X_{n} / d q_{S}$ or $d Y_{n} / d q_{S}$, while the corresponding sensitivities (minimal detectable charge $\delta q_{S}$ for a small measurement bandwidth $\Delta f$ ) are

$$
\delta q_{S, X n}=\frac{\sqrt{S_{X n} \Delta f}}{\left|d X_{n} / d q_{S}\right|}, \quad \delta q_{S, Y n}=\frac{\sqrt{S_{Y n} \Delta f}}{\left|d Y_{n} / d q_{S}\right|} .
$$

Numerical calculations show that in the usual case $\omega \approx \omega_{0}$ thus defined RF-SET sensitivity for overtones $(n \geq 2)$ can be comparable to the sensitivity using the carrier frequency $(n=1)$. However, because of relatively small amplitude of reflected overtones in this case, their monitoring is impractical, and so we mainly consider monitoring of $X_{1}$ and $Y_{1}$ which are referred below as $X$ and $Y$.

Monitoring both quadratures $X$ and $Y$ simultaneously, one can improve the sensitivity compared with monitoring of only one quadrature. It is easy to show that the resulting sensitivity can be obtained as the optimization over angle $\varphi$ of the sensitivity corresponding to monitoring the linear combination $X^{*}=X \cos \varphi+Y \sin \varphi$ (experimentally, this is just a phase shift in the homodyne detector; notice that the contribution $-V_{\text {in }} \cos \omega t$ is noiseless by assumption). The optimum sensitivity is achieved at $\tan \varphi=\left(S_{X} d Y / d q_{S}-S_{X Y} d X / d q_{S}\right) /\left(S_{Y} d X / d q_{S}-\right.$ $\left.S_{X Y} d Y / d q_{S}\right)$ and the resulting sensitivity is

$$
\frac{\delta q_{S, X^{*}}}{\sqrt{\Delta f}}=\left[\frac{S_{X} S_{Y}-S_{X Y}^{2}}{S_{Y}\left(\frac{d X}{d q_{S}}\right)^{2}+S_{X}\left(\frac{d Y}{d q_{S}}\right)^{2}-2 S_{X Y} \frac{d X}{d q_{S}} \frac{d Y}{d q_{S}}}\right]^{1 / 2},
$$

that can be rewritten $\operatorname{as}^{30}$

$\frac{\delta q_{S, X^{*}}}{\sqrt{\Delta f}}=\left[\frac{1-K^{2}}{\left(\delta q_{S, X}\right)^{-2}+\left(\delta q_{S, Y}\right)^{-2}-2 K / \delta q_{S, X} \delta q_{S, Y}}\right]^{1 / 2}$,

where $K \equiv S_{X Y} / \sqrt{S_{X} S_{Y}} \operatorname{sign}\left[\left(d X / d q_{S}\right)\left(d Y / d q_{S}\right)\right]$ is the noise correlation factor $\left[S_{X Y}\right.$ is a real magnitude because we consider only low-frequency sensitivity; for finite frequency $S_{X Y}$ in Eq. (38) should be replaced by $\left.\operatorname{Re} S_{X Y}\right]$. Notice that the response optimization for $X^{*}$ monitoring is achieved at different phase shift: $\tan \varphi=\left(d Y / d q_{S}\right) /\left(d X / d q_{S}\right)$ that leads to the response $d X^{*} / d q_{S}=\left[\left(d X / d q_{S}\right)^{2}+\left(d Y / d q_{S}\right)^{2}\right]^{1 / 2}$.

Let us also consider the case when the reflected wave is monitored by simple rectification. Assuming for simplicity the monitoring of only the first harmonic amplitude $A_{1}=\left[\left(X-V_{i n}\right)^{2}+Y^{2}\right]^{1 / 2}$ (overtones are filtered out), the RF-SET sensitivity can be calculated as

$$
\frac{\delta q_{S, A 1}}{\sqrt{\Delta f}}=\frac{\left[\left(X-V_{i n}\right)^{2} S_{X}+Y^{2} S_{Y}+2\left(X-V_{i n}\right) Y S_{X Y}\right]^{1 / 2}}{\left|\left(X-V_{i n}\right) d X / d q_{S}+Y d Y / d q_{S}\right|}
$$

while the response is obviously $d A_{1} / d q_{S}=[(X-$ $\left.\left.V_{i n}\right) d X / d q_{S}+Y d Y / d q_{S}\right] / A_{1}$. The formulas for monitoring of $n$th overtone are similar, except $V_{i n}$ does not contribute to the amplitude $A_{n}$.

In the most interesting for practice case $\omega=\omega_{0}$ the magnitude of $Y$ is small in comparison with $X$ for $Q \gg 1$ [see Eqs. (25)-(26)], and the correlation factor $K$ also vanishes [because $d_{1}=0$ and the SET bias voltage contains mostly sin-component - see Eq. (27)]. Then Eq. (39) for the best homodyne detection practically coincides with Eq. (40) for rectification and reduces to the sensitivity $\delta q_{S, X} / \sqrt{\Delta f}=\sqrt{S_{X}} /\left(d X / d q_{S}\right)$ for monitoring of $X$ component only (similarly, the formulas for response also practically coincide). Because of that, the numerical results for RF-SET response and sensitivity in the case $\omega=\omega_{0}$ will assume monitoring of $X$ quadrature.

Since the increments of the measured charge $q_{S}$ and the effective SET charge $q_{0}$ are related by a constant factor,

$$
\delta q_{S}=\delta q_{0}\left(1+C_{S} / C_{g}\right),
$$

the RF-SET response and sensitivity in respect to $q_{S}$ and in respect to $q_{0}$ differ by the factor $1+C_{S} / C_{g}$. All our numerical results will be in terms of $q_{0}$ measurement, so for $X$ monitoring we will use the derivative $d X / d q_{0}$ as a measure of the RF-SET response and the magnitude

$$
\frac{\delta q_{0}}{\sqrt{\Delta f}}=\frac{\sqrt{S_{X}}}{\left|d X / d q_{0}\right|}
$$

as a measure of the sensitivity.

\section{NUMERICAL RESULTS}

We have studied numerically the dependence of the RF-SET response and sensitivity on various parameters, which include "fixed" parameters (which cannot be easily changed in an experiment) and the parameters of the operation point. The fixed parameters are: effective junction capacitances of the SET $C_{1}$ and $C_{2}$ (we assume $C_{1}=C_{2}$ unless mentioned otherwise), resistances $R_{1}$ and $R_{2}$ (we always assume $R_{1}=R_{2}$ ), temperature $T$, cable wave impedance $R_{0}$ (we always assume $R_{0}=50 \Omega$ ), the 

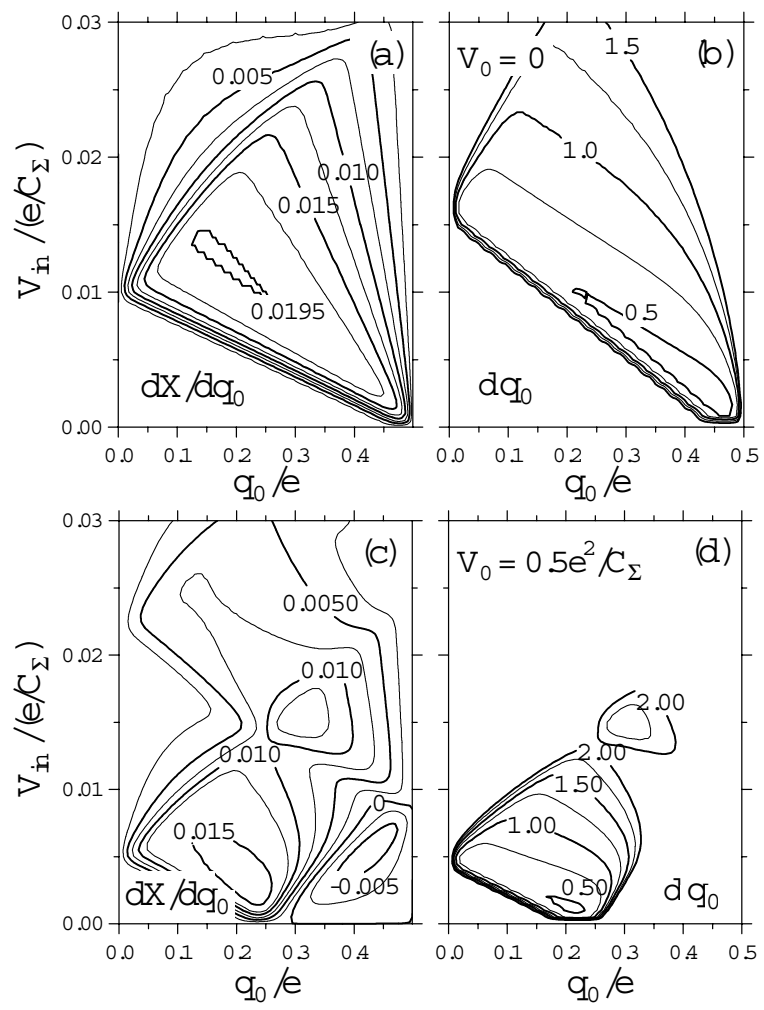

FIG. 2. Contour plots of (a, c) the RF-SET response $d X / d q_{0}$ (in units $C_{\Sigma}^{-1}$ ) and (b, d) the noise-limited sensitivity $\delta q_{0} / \sqrt{\Delta f}$ (in units $e \sqrt{R_{\Sigma} C_{\Sigma}}$ ) on the plane of the SET background charge $q_{0}$ and the amplitude $V_{i n}$ of incoming $\mathrm{rf}$ wave for $Q=50, T=0.01 e^{2} / C_{\Sigma}, R_{\Sigma} / R_{0}=2000$, and $\omega=\omega_{0}$. $V_{0}=0$ for panels (a) and (b), and $V_{0}=0.5 e^{2} / C_{\Sigma}$ for panels (c) and (d).

tank circuit frequency $\omega_{0}=1 / \sqrt{L_{T} C_{T}}$, and the $Q$-factor $Q=\sqrt{L_{T} / C_{T}} / R_{0}$. The operating point parameters are the effective SET background charge $q_{0}$, dc bias voltage $V_{0}$, amplitude $V_{i n}$ of the incident wave, and its frequency $\omega$ (in most cases we assume $\omega=\omega_{0}$ ).

We use the SET parameters for normalization, so that a natural unit for temperature is $e^{2} / C_{\Sigma}$ (where $C_{\Sigma}=$ $C_{1}+C_{2}$ ), the voltage unit is $e / C_{\Sigma}$, the RF-SET response $d X / d q_{0}$ (or $\left.d Y / d q_{0}\right)$ can be measured in units $1 / C_{\Sigma}$, and the unit for sensitivity $\delta q_{0} / \sqrt{\Delta f}$ is $e\left(R_{\Sigma} C_{\Sigma}\right)^{1 / 2}$ (where $\left.R_{\Sigma}=R_{1}+R_{2}\right)$. Notice that all considered magnitudes have a simple scaling with $C_{\Sigma}$; however, there is no simple scaling with $R_{\Sigma}$ because of the dimensionless parameter $R_{\Sigma} / R_{0}$.

\section{Operation point optimization}

Figure 2(a) shows the RF-SET response $d X / d q_{0}$ on the plane of operating point parameters $q_{0}$ and $V_{i n}$ for the case $Q=50, R_{\Sigma} / R_{0}=2000$ (i.e. $R_{\Sigma}=100 \mathrm{k} \Omega$ ), $V_{0}=0, \omega=\omega_{0}$, and $T=0.01 e^{2} / C_{\Sigma}$ (the SET is symmetric, $\left.C_{1}=C_{2}, R_{1}=R_{2}\right)$. Figure $2(\mathrm{c})$ is similar, except
$V_{0}=0.5 e^{2} / C_{\Sigma}$. We do not show $d Y / d q_{0}$ because it is practically vanishing. Because of the same reason, the sensitivity $\delta q_{0} / \sqrt{\Delta f}$ shown in Figs. 2(b) and 2(d) is calculated using only quadrature $X$.

Both the response and sensitivity are obviously poor when the amplitude of oscillations at the SET is below the Coulomb blockade threshold $V_{t}$ [this happens at $V_{\text {in }}<V_{t} / 2 Q$ where $V_{t}=\left(e / C_{\Sigma}\right)\left(1-2 q_{0} / e\right)$, and corresponds to the triangles in the lower left corners of Figs. $2(\mathrm{a})-(\mathrm{d})$ ], and they are also poor when $V_{i n}$ is much larger than this condition (notice that better response corresponds to larger $d X / d q_{0}$, while better sensitivity corresponds to smaller $\delta q_{0}$ ). Even though the regions of relatively good response and sensitivity are similar, the maximum response and optimum sensitivity are achieved at quite different points in the $V_{i n}-q_{0}$ plane. In particular, the amplitude $V_{i n}$ of the incoming wave is significantly larger for maximum response, than for best sensitivity.

In the present-day experiments, maximization of response [Figs. 2(a) and 2(c)] is still of the major importance, because the noise from the next amplifying stage is still significant [if a large constant noise had been added to $S_{X}$ in Eq. (42), then the sensitivity would be mainly determined by the denominator $\left.d X / d q_{0}\right]$. However, if the amplifier noise is small compared to the contribution from the SET shot noise, then the best operation point should optimize the shot-noise-limited sensitivity [Figs. 2 (b) and 2(d)]. In the case of comparable contributions from two noises, we have a trade-off between two regimes. In this paper we will concentrate on the analysis of the maximum response (MR) mode and optimized sensitivity (OS) mode, keeping in mind that experimentally optimal regime is somewhere in between, depending on the amplifier noise.

Figure 3 shows dependence of the response $d X / d q_{0}$ and sensitivity $\delta q_{0} / \sqrt{\Delta f}$ on the dc bias voltage $V_{0}$ in the MR and OS modes (optimizations are over $V_{i n}$ and $q_{0}$ ). Several curves on each plot are for different $Q$-factors: $Q=10,30,50,70$, and 90, while other parameters are similar to the parameters of Fig. 2. One can see that the best response in both MR and OS regimes as well as the best sensitivity in OS mode are achieved at $V_{0}=0$. This is because both positive and negative branches of the symmetric SET $I-V$ curve (see Fig. 10 in Appendix) contribute equally at $V_{0}=0$, and therefore the signal is maximal.

In the MR mode at $V_{0}=0$ the optimum background charge [see Fig. 2(a)] is about $q_{0} \approx 0.15 e$ (so the Coulomb blockade threshold $V_{t}$ is about $0.7 e / C_{\Sigma}$ ) while the optimum amplitude $A_{b}$ of the SET bias $V_{b}$ oscillations is about $1.1 \mathrm{e} / C_{\Sigma}$ (these numbers have only weak dependence on $Q$, while the optimum $V_{i n}$ obviously depends on $Q$ quite significantly). When $V_{0}$ starts to increase, it becomes advantageous to increase $q_{0}$ (so $V_{t}$ decreases) while $A_{b}$ stays approximately constant, so that both positive and negative branches of the SET $I-V$ still contribute to the response. However, since these branches cannot both contribute in the optimal way, the response decreases 

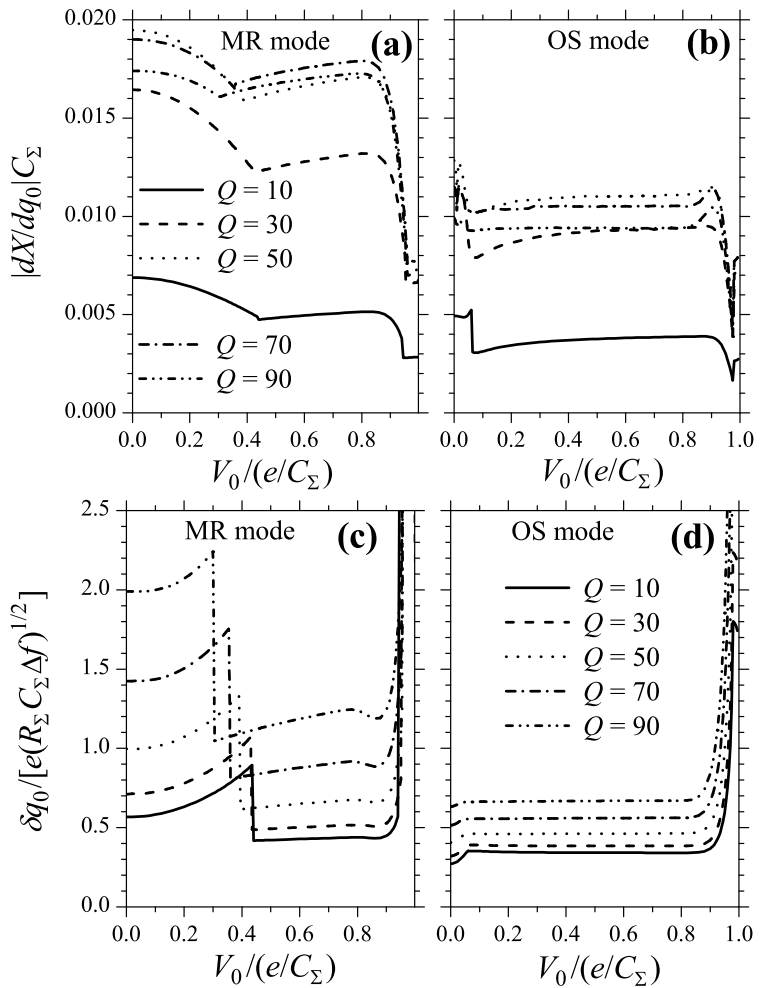

FIG. 3. Dependence of (a)-(b) the RF-SET response $d X / d q_{0}$ and (c)-(d) sensitivity $\delta q_{0} / \sqrt{\Delta f}$ on the dc bias voltage $V_{0}$ for several values of the tank circuit $Q$-factor. $V_{\text {in }}$ and $q_{0}$ are optimized for either maximum response [MR mode, panels (a) and (c)] or optimal sensitivity [OS mode, panels (b) and (d)], while other parameters are: $T=0.01 e^{2} / C_{\Sigma}$, $R_{\Sigma} / R_{0}=2000$, and $\omega=\omega_{0}$.

with $V_{0}$ [see Fig. 3(a)]. For large enough $V_{0}$ it becomes preferable to use only one (positive) branch [this regime corresponds to the lower maximum in Fig. 2(c), while the upper maximum corresponds to the two-branch regime]; then the optimal wave amplitude drops to $A_{b} \simeq 0.2 e / C_{\Sigma}$ and the optimal $q_{0}$ corresponds to $V_{t}$ slightly above $V_{0}$ (by about $0.03 e / C_{\Sigma}$ ). This change causes the kinks on the response curves in Fig. 3(a) and jumps down on the sensitivity curves in Fig. 3(c). Notice that in the MR mode the sensitivity at $V_{0} \simeq 0.5 e / C_{\Sigma}$ is better than at $V_{0}=0$.

In the OS regime the optimal amplitude is significantly less than in the MR regime. It depends mainly on temperature, and for parameters of Fig. 3 the amplitude $A_{b}$ at the SET is typically between 0.08 and 0.1 in units of $e / C_{\Sigma}$. The "above blockade" voltage $V_{0}+A_{b}-V_{t}$ is few times smaller than $A_{b}$ and is comparable to the temperature. The best sensitivity is achieved at $V_{0}=0$ when both branches participate, while at large enough $V_{0}$ when it becomes preferable to use only one branch, both sensitivity and response practically do not depend on $V_{0}$ [see Figs. 3(b) and 3(d)].
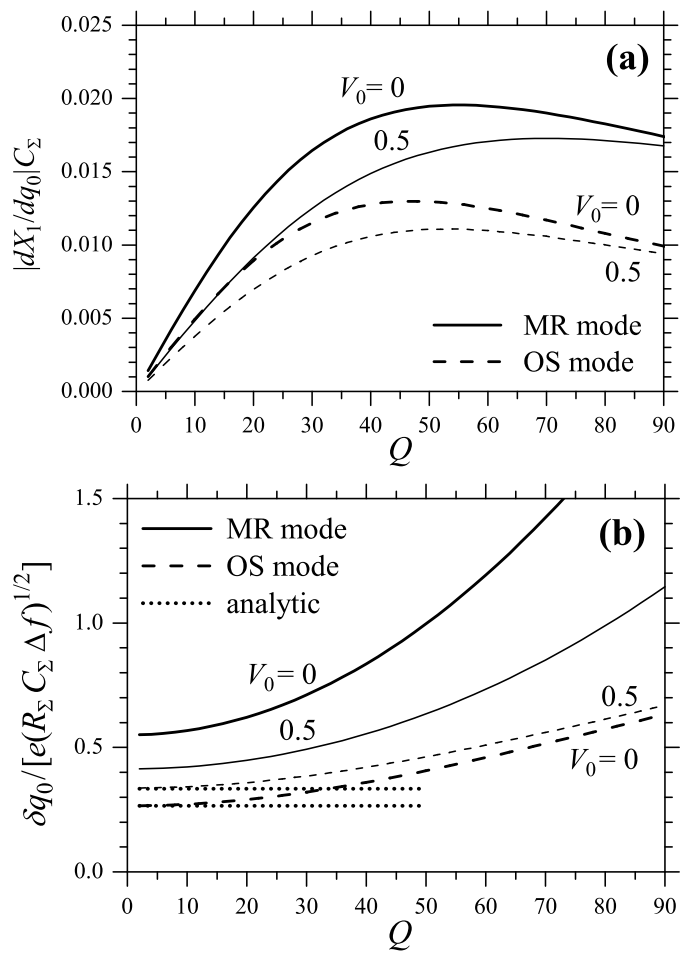

FIG. 4. (a) RF-SET response and (b) sensitivity as functions of the $Q$-factor in the maximum response and optimal sensitivity modes. $T=0.01 e^{2} / C_{\Sigma}, R_{\Sigma} / R_{0}=2000$, and $\omega=\omega_{0}$. Notice monotonic worsening of the sensitivity with $Q$. The horizontal dotted lines in (b) show the low- $Q$ low- $T$ results $^{30}$ corresponding to Eqs. (43) and (44).

\section{Dependence on $Q$-factor}

The dependence on $Q$-factor is summarized in Fig. 4, which shows the response and sensitivity in the MR and OS modes for $T=0.01 e^{2} / C_{\Sigma}, R_{\Sigma} / R_{0}=2000$, and $\omega=\omega_{0}$. The results are presented for $V_{0}=0$ (thick lines), which provides the best MR and OS response and best OS sensitivity, and also for $V_{0}=0.5 e / C_{\Sigma}$ (thin lines), which is a typical value for the case when only one branch of the SET $I-V$ is involved. One can see that the response $d X / d q_{0}$ grows linearly with $Q$ at small $Q$ [see Eq. (11)] and reaches the maximum at $Q$ around 50 (this number is somewhat different for different regimes; for example it is almost 70 for the thin solid line), which is close to the crude theoretical estimate $\sqrt{R_{\Sigma} / R_{0}} \simeq 45$ for the impedance matching. However, unlike in the linear model, this maximum does not correspond to the exact impedance matching. For example, the impedance matching (minimum of reflection) occurs at $Q \simeq 100$ for the upper curve in Fig. 4(a) and at $Q \simeq 80$ for the curve second from the top, while for two lower curves (OS mode) it does not occur at all in a reasonable range of $Q$.

In contrast to the response behavior, the RF-SET sensitivity monotonically worsens with increase of $Q$. Qualitatively, this happens because the noise $S_{X}$ has $Q^{2}$ scal- 
ing [see Eq. (35)], while the response $d X / d q_{0}$ has slower than $Q$ dependence [see Eq. (11)]. This simple analysis predicts the parabolic dependence $\delta q_{0} \propto 1+Q^{2} /\left(R_{d} / R_{0}\right)$, which crudely fits the curves in Fig. 4(b) using $R_{d} / R_{0}$ about 1.5-3 times larger than $R_{\Sigma} / R_{0}$, though the curves in Fig. 4(b) actually have slower than parabolic dependences at large $Q$.

Comparing the MR and OS modes at $Q=30$ (just some typical number) and $V_{0}=0$, we see that the MR regime provides about $40 \%$ larger response, while the OS regime provides about twice better sensitivity. Even though these numbers depend significantly on the temperature and also depend on $Q$ and $R_{\Sigma} / R_{0}$, they show that the results for the MR and OS regimes are not too much different (not by an order of magnitude).

Comparison of the cases $V_{0}=0$ and $V_{0}=0.5 \mathrm{e} / C_{\Sigma}$ shows that for the MR and OS response as well as for the OS sensitivity there is no much difference between these two cases, and the relative difference decreases with $Q$. In contrast, the difference between MR sensitivity at $V_{0}=0$ and at $V_{0}=0.5 e / C_{\Sigma}$ grows with $Q$ and can become significant.

The low- $Q$ limit of the OS sensitivity for $V_{0}=0$ is well described by the formula ${ }^{30}$ (which also assumes $T \ll$ $\left.e^{2} / C_{\Sigma}\right)$

$$
\delta q_{0} / \sqrt{\Delta f} \simeq 2.65 e\left(R_{\Sigma} C_{\Sigma}\right)^{1 / 2}\left(T C_{\Sigma} / e^{2}\right)^{1 / 2}
$$

[we do not use a shorter formula $2.65 C_{\Sigma}\left(R_{\Sigma} T\right)^{1 / 2}$ to emphasize natural normalizations], and similar limit for $V_{0}=0.5 e / C_{\Sigma}$ (one-branch case) is close to ${ }^{30}$

$$
\delta q_{0} / \sqrt{\Delta f} \simeq 3.34 e\left(R_{\Sigma} C_{\Sigma}\right)^{1 / 2}\left(T C_{\Sigma} / e^{2}\right)^{1 / 2}
$$

[see Fig. 4(b)]. However, the theory of Ref. ${ }^{30}$ which assumes $Q \ll \sqrt{R_{\Sigma} / R_{0}}$, is not able to describe significant change of the sensitivity with $Q$ in Fig. 4(b). One can see that this dependence is even more significant in the MR mode.

\section{Dependence on temperature and SET resistance}

The numerical results on temperature dependence are shown in Fig. 5 for $R_{\Sigma} / R_{0}=2000$ and $\omega=\omega_{0}$. Similar to Fig. 3 we show on four panels the response $d X / d q_{0}$ and corresponding sensitivity in the MR and OS modes (the optimal dc bias value $V_{0}=0$ is used). It is important to notice that in the MR mode both response and sensitivity almost do not depend on temperature at $T<0.03 e^{2} / C_{\Sigma}$, and the RF-SET performance is still OK at temperatures $\sim 0.1 e^{2} / C_{\Sigma}$ (response and sensitivity change less than twice compared to zero-temperature case). In the OS regime the response also has a very weak temperature dependence at $T<0.05 e^{2} / C_{\Sigma}$; however, the sensitivity is strongly temperature-dependent. The low$Q$ OS sensitivity can be accurately described by Eq. (43)
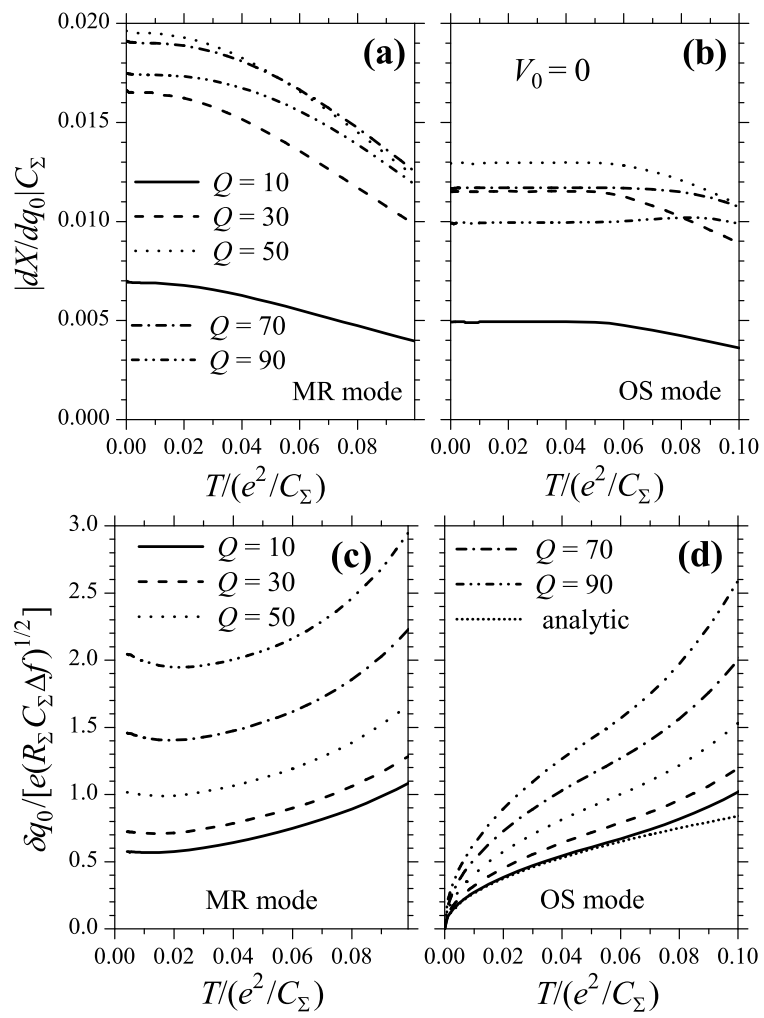

FIG. 5. Dependence of (a)-(b) RF-SET response and (c)-(d) sensitivity on temperature $T$ in the MR and OS regimes for several $Q$-factors. $V_{0}=0, R_{\Sigma} / R_{0}=2000$, and $\omega=\omega_{0}$. Lowest dotted line in (d) is Eq. (43).

up to temperatures $\sim 0.05 e^{2} / C_{\Sigma}$, [see the lowest dotted line in Fig. 5(d)], and the curves for large $Q$-factors also follow the scaling $\delta q_{0} / \sqrt{\Delta f} \propto T^{1 / 2}$ at temperatures $T<0.05 e^{2} / C_{\Sigma}$. In the orthodox theory this $T^{1 / 2}$ dependence is valid even at very small temperatures leading to infinitely good sensitivity $\left(\delta q_{0} / \sqrt{\Delta f} \rightarrow 0\right)$; however, in reality the neglected contribution of cotunneling processes becomes significant in the OS regime at small $T$ that changes the formalism. ${ }^{22,36}$ Comparing the results for MR and OS modes, we notice that while they are significantly different at low temperatures, the difference decreases with temperature, so that at $T \sim 0.1 e^{2} / C_{\Sigma}$ the MR and OS results are already quite similar.

Figure 6 shows the response and sensitivity dependence on the total SET junction resistance $R_{\Sigma}$ for $T=$ $0.01 e^{2} / C_{\Sigma}, V_{0}=0$, and $\omega=\omega_{0}$ (we show results only for $R_{\Sigma} / R_{0}>500$, because at $R_{\Sigma}<25 \mathrm{k} \Omega$ the theory is too inaccurate due to neglected cotunneling processes). In the case ${ }^{30} Q \ll \sqrt{R_{\Sigma} / R_{0}}$ the response $d X / d q_{0}$ scales as $R_{\Sigma}^{-1}$ [see Eq. (23)] and the sensitivity $\delta q_{0} / \sqrt{\Delta f}$ scales as $R_{\Sigma}^{1 / 2}$ [see Eq. 43]. Correspondingly, the solid lines $(Q=10)$ in Figs. 6(a) and (b) are practically straight lines passing through the origin, and in Figs. 6(c) and (d) the solid lines are practically horizontal [the level determined by Eq. (43) is shown in Fig. 6(d) by the lowest 

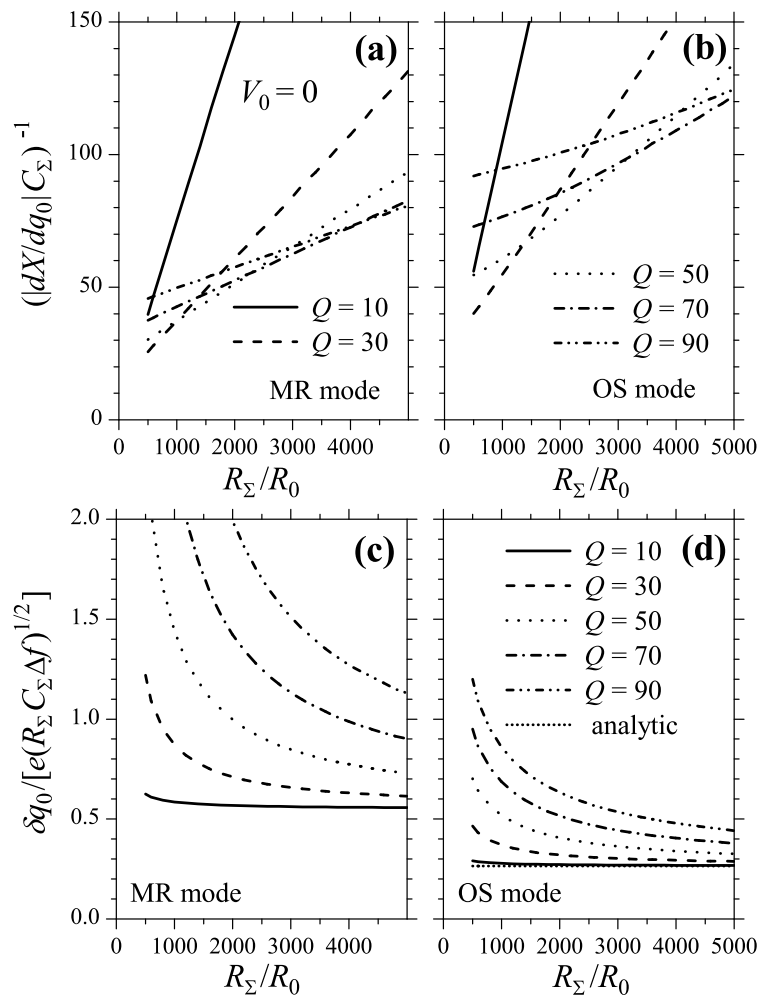

FIG. 6. (a)-(b) Inverse response and (c)-(d) sensitivity in the MR and OS regimes as functions of the SET resistance $R_{\Sigma}$ for several $Q$-factors at $T=0.01, V_{0}=0$, and $\omega=\omega_{0}$.

dotted line, which fits well the solid line]. The dependence on $R_{\Sigma}$ becomes nontrivial when $R_{\Sigma} / R_{0}$ is comparable to $Q^{2}$. It is interesting to notice that the inverse response remains a practically linear function of $R_{\Sigma}$ even for large $Q$, as seen in Figs. 6(a) and (b) (the slope of the lines decreases with $Q$ while the offset increases). In particular, this means that in the orthodox model the RF-SET response continues to increase with the SET resistance decrease even when the matching condition is overshot. Decrease of $R_{\Sigma}$ close or beyond the matching condition, worsens the sensitivity in comparison with scaling $\delta q_{0} / \sqrt{\Delta f} \propto R_{\Sigma}^{1 / 2}$ [see Figs. 6(c) and (d)]. In the MR mode the sensitivity worsens with decrease of $R_{\Sigma}$ even in absolute units $(e / \sqrt{\mathrm{Hz}})$ when the matching condition is sufficiently overshot, while in the OS mode the sensitivity still improves with decrease of $R_{\Sigma}$ in absolute units, in spite of worsening compared to $R_{\Sigma}^{1 / 2}$ scaling.

\section{Effect of asymmetric biasing $\left(C_{1} \neq C_{2}\right)$}

Now let us discuss the effect of asymmetric effective capacitances of the SET junctions, $C_{1} \neq C_{2}$. Even when the physical junction capacitances are equal, $C_{1 j}=C_{2 j}$, the effective capacitances $C_{1}$ and $C_{2}$ can be significantly different when the gate capacitance $C_{g}$ is comparable to junction capacitances. In our model shown in Fig. 1 this
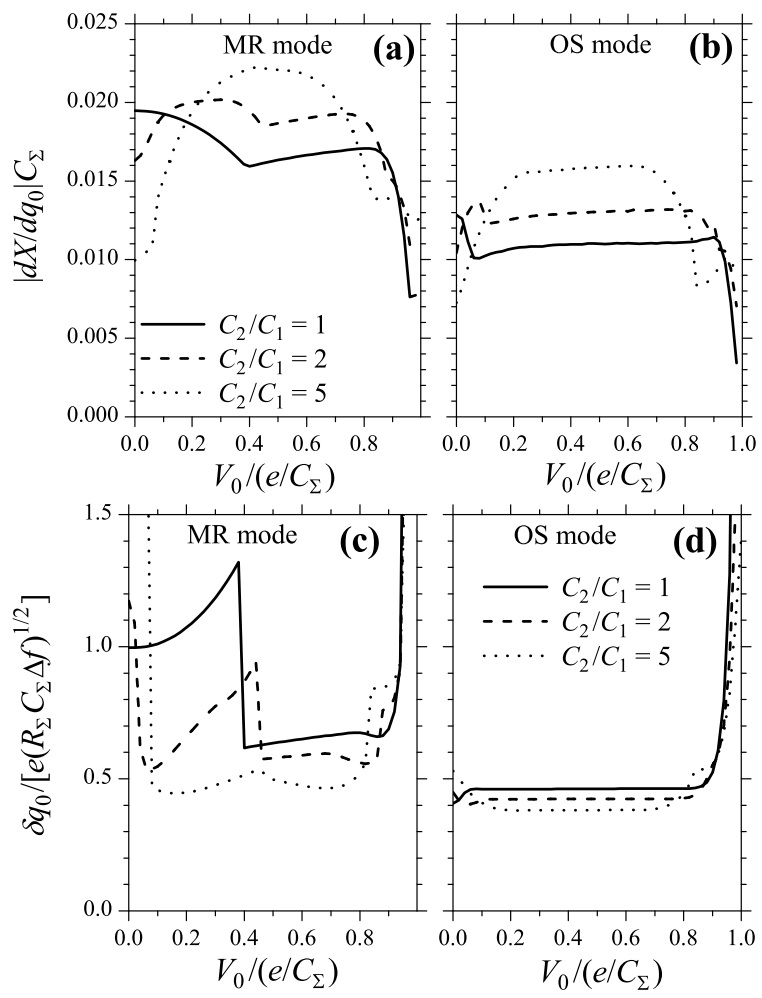

FIG. 7. (a)-(b) Dependence of the RF-SET response and (c)-(d) sensitivity on the dc bias voltage $V_{0}$ for several values of the effective asymetry of the SET capacitances. $T=0.01 e^{2} / C_{\Sigma}, Q=50, R_{\Sigma} / R_{0}=2000$, and $\omega=\omega_{0}$. Notice that the SET asymmetry ("asymmetric biasing") slightly improves the RF-SET performance.

happens due to asymmetry of signal source capacitances, $C_{S 1} \neq C_{S 2}$ [see Eqs. (1) and (2)]; in RF-SET experiments this effect is called asymmetric rf biasing of the SET. ${ }^{11}$ For the conventional SET setup, the biasing asymmetry is not important at all, because of the formal SET equivalence under transformation ${ }^{37}$

$$
C_{1} \rightarrow C_{1}+\Delta C, C_{2} \rightarrow C_{2}-\Delta C, q_{0} \rightarrow q_{0}-V_{b} \Delta C
$$

for arbitrary $\Delta C$, that means that the asymmetric biasing (different $C_{1}$ and $C_{2}$ ) can be simply corrected by the background charge shift. However, for the RF-SET there is no simple equivalence because the bias voltage $V_{b}$ changes in time. (Effective capacitance asymmetry can still be controlled by addition of the extra rf signal to the SET gate.) Therefore it is interesting to find if the asymmetric biasing $\left(C_{1} \neq C_{2}\right)$ is better or worse than the symmetric case $\left(C_{1}=C_{2}\right)$.

Figure 7 shows the $V_{0}$ dependence of the RF-SET response and sensitivity in the MR and OS modes for several ratios $C_{1} / C_{2}$ (with fixed total capacitance $C_{1}+C_{2}$ ). One can see that in the asymmetric cases the best MR response and OS sensitivity are achieved at nonzero $V_{0}$ and are typically better than the corresponding values for the symmetric case. Therefore, the asymmetric biasing is actually preferable (that eases the concern about 
this issue discussed in Ref. ${ }^{11}$ ); however, the advantage is rather minor. In particular, the OS sensitivity in the asymmetric case is still limited by Eq. (44); therefore the possible improvement is less than the sensitivity decline due to high $Q$-factor [see Fig. 4(b)], and even less because of the difference between Eqs. (43) and (44).

\section{Carrier frequency detuning from the resonance}

So far we have considered the resonant case $\omega=\omega_{0}$. [Actually, the exact resonance is at the frequency $\omega=$ $\omega_{0}\left(1-1 / 2 Q_{S E T}^{2}\right)$ which is very close to $\omega_{0}$.] In this case the quadrature component $X$ is much larger than $Y$ (at $Q \gg 1$ ) and therefore the RF-SET response and sensitivity in respect to monitoring $X$ quadrature practically coincide with that for monitoring the reflected wave amplitude $A$ (we denote with $A$ the amplitude $A_{1}$ of the first harmonic) or monitoring the optimized phase-shifted combination $X^{*}=X \cos \varphi+Y \sin \varphi$. The detuning of $\omega$ from the resonant frequency $\omega_{0}$ leads to a significant magnitude of $Y$ quadrature, and so to different results for different ways of reflected wave monitoring.

For a small frequency detuning $\Delta \omega=\omega-\omega_{0}$ the simple linear analysis using Eq. (11) predicts the nonmonotonic shape of the $X$-response frequency dependence with zeros at $\omega / \omega_{0}=1 \pm 1 / 2 Q_{L}$ and with the full width at half height $(\mathrm{FWHH})$ equal to $0.53 \omega_{0} / Q_{L}$; while for the amplitude monitoring it predicts FWHH of $\sqrt{3} \omega_{0} / Q_{L}$. [Eq. (8) which assumes both $R_{d}$ and $V_{i n}$ being frequency-independent, gives the response $\mathrm{FWHH}$ equal to $(\sqrt{5}-2)^{1 / 2} \omega_{0} / Q_{L}$ and $\omega_{0} / Q_{L}$ for $X$ and $A$ monitoring, respectively]. In reality the situation is more complex because even neglecting the nonlinear contributions, the effective SET resistance $R_{d}$ (and therefore $Q_{L}$ ) depends on $V_{b}(t)$ amplitude, which depends itself on $\omega, R_{d}$, and $V_{i n}$. Moreover, the optimized point in the $V_{i n}-q_{0}$ plane is also frequency-dependent.

Figure 8 shows the numerical results for the frequency dependence (in the vicinity of the resonance) of the optimized response and sensitivity. Each panel shows four curves, which correspond to monitoring the components $X$ (thick solid line), $Y$ (dashed), optimized phase-shifted combination $X^{*}$ (thin solid), and amplitude $A$ (dotted line). At each frequency we perform optimizations of the response (MR mode) and sensitivity (OS mode) over $V_{i n}-q_{0}$ plane for all four monitored magnitudes, so the optimizations are different for different curves. The $Q$ factor is equal to 50, which is close to the estimate of the impedance-matching value (see also Fig. 4); however, instead of the naively expected value $Q_{L} \simeq Q / 2=25$ for the loaded $Q$-factor, it is $Q_{L}=40$ in the $\mathrm{MR}$ mode since $Q_{S E T}=199$ (these values are calculated at $\omega=\omega_{0}$ using effective SET resistance). The shape of the $X$-response dependence in panel (a) is close to the prediction of Eq. (11) using $Q_{L}=40$, though the minima do not reach zero and the curve beyond the minima is shifted up. The
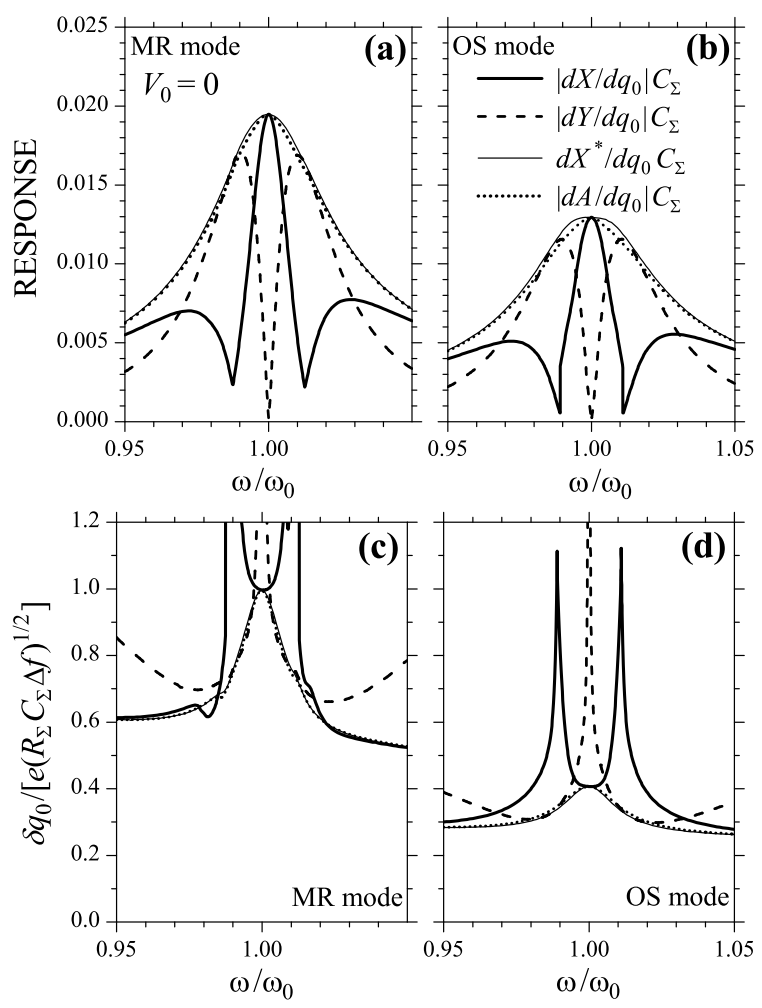

FIG. 8. Frequency dependence of the (a)-(b) RF-SET response and (c)-(d) sensitivity in the MR and OS modes. Each panel shows four curves corresponding to monitoring of $X$ quadrature (thick solid line), $Y$ quadrature (dashed), their optimized phase-shifted combination $X^{*}$ (thin solid), and the first harmonic amplitude $A$ (dotted). $Q=50$, $T=0.01 e^{2} / C_{\Sigma}, R_{\Sigma} / R_{0}=2000, V_{0}=0$.

MR $Y$-response is practically zero at $\omega=\omega_{0}$ (since $Y$ vanishes); however, it becomes comparable to the resonant $X$-response at some frequency detuning; the overall shape is close to the prediction of Eq. (11), but the maxima are about $15 \%$ higher. The MR $A$-response curve is about $50 \%$ wider than the prediction of the linear theory (using $Q_{L}=40$ ) and is very close to the curve for monitoring optimal phase-shifted quadrature $X^{*}$. As expected, the $A$ - and $X^{*}$-responses are better than $X$ and $Y$-responses at finite detuning (for both MR and OS modes).

The sensitivity (both OS and MR) for $X^{*}$ or $A$ monitoring at finite detuning is also better then for $X$ or $Y$ monitoring. An interesting observation is that while the RF-SET response decreases with detuning, the sensitivity slightly improves with detuning for $X^{*}$ and $A$ monitoring. This effect is similar to the sensitivity improvement with the decrease of $Q$-factor (see Fig. 4). 

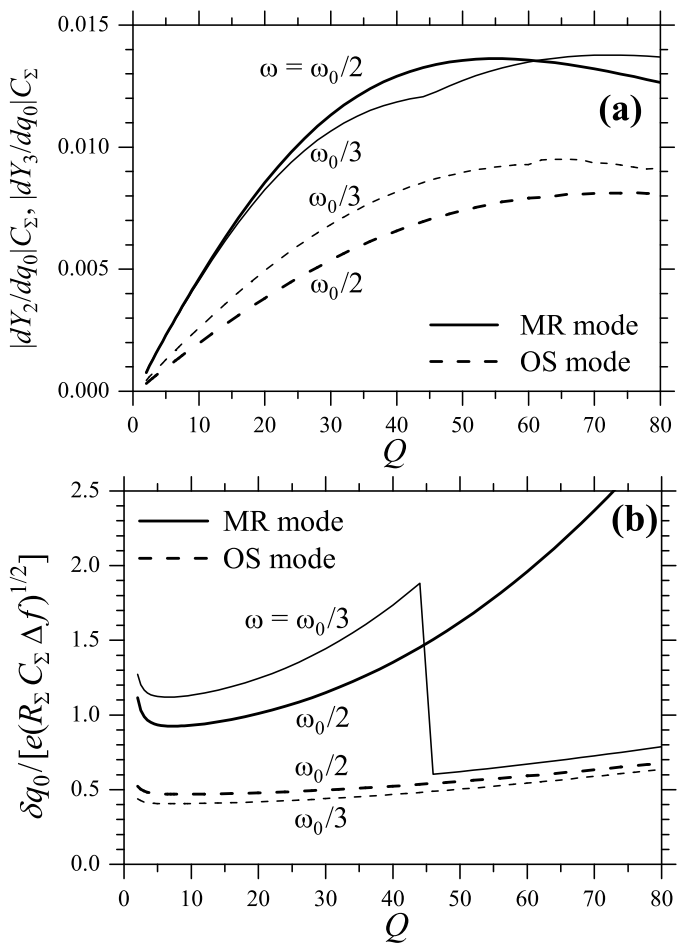

FIG. 9. (a) RF-SET response and (b) sensitivity in the regimes when the second or third overtone of the incident $\mathrm{rf}$ wave is in resonance with the tank circuit. $T=0.01 e^{2} / C_{\Sigma}$, $R_{\Sigma} / R_{0}=2000 ; V_{0}=0.5 e / C_{\Sigma}$ for $\omega=\omega_{0} / 2$ and $V_{0}=0$ for $\omega=\omega_{0} / 3$.

\section{Monitoring of resonant overtone}

In experiments the incoming rf wave is usually tuned close to the resonance with the tank circuit; in this case the contribution of overtones in the reflected $\mathrm{rf}$ wave is small in spite of significant SET $I-V$ nonlinearity (the SET nonlinearity has been recently used ${ }^{38}$ for rf mixing). However, if the $n$th overtone is in resonance, $\omega \approx \omega_{0} / n$, then the reflected wave may have a significant contribution from this overtone, and the RF-SET operation can be based on monitoring this overtone. ${ }^{35}$ The use of different frequencies for the incident and reflected waves may be advantageous for some applications. Also, it may be useful to have the absence of the monitored reflected wave when the SET is off (no current), while in the conventional regime this case corresponds to the largest reflected power. One more possible advantage is somewhat easier control of the amplitude of the SET bias voltage oscillations, since now it is more directly related to the incident amplitude $V_{i n}$, while in the usual regime the relation depends on $Q_{L}$ [see Eq. (27)] which varies with operating point. (The disadvantage is that the incident amplitude $V_{\text {in }}$ should be much larger than in usual regime that may lead to heating problems.)

Figure 9 shows the RF-SET response and sensitivity for $\omega=\omega_{0} / 2$ and $\omega=\omega_{0} / 3$, in respect to monitoring $Y_{2}$ and $Y_{3}$, correspondingly (the $X$-quadratures are small).
We use $V_{0}=0$ in the case $\omega=\omega_{0} / 3$ and $V_{0}=0.5 e / C_{\Sigma}$ in the case $\omega=\omega_{0} / 2$ [for $V_{0}=0$ there is no second overtone because of the $I-V$ curve symmetry even at nonzero $q_{0}$ - see Fig. 10(a) and Eq. (17)]. As seen from Fig. 9, the MR responses and OS sensitivities in the two cases (second and third resonant overtone) are quite similar, that is related to a strong nonlinearity of the SET $I-V$ curve. Moreover, comparing Figs. 4 and 9 (the parameters are the same) we see that the RF-SET performance in the regime of a resonant overtone is comparable to the performance in the conventional regime $\omega=\omega_{0}$ (the MR response and OS sensitivity are worse by only about 1.5 times). Combined with the advantages discussed above, this makes the regime of resonant overtone potentially useful in experiments.

\section{CONCLUSION}

In this paper we have developed the formalism for the calculation of the response and shot-noise-limited sensitivity of the normal-metal RF-SET, extending the theory of Ref. ${ }^{30}$ to the case of arbitrary large $Q$-factor of the tank circuit and arbitrary frequency of the incident $\mathrm{rf}$ wave. The formalism has been used to analyze numerically the dependence of the RF-SET response and sensitivity on the operation parameters.

We have mainly studied two operation modes, optimized over the rf wave amplitude and the SET background charge, corresponding to either maximum response (MR mode) or optimum sensitivity (OS mode). The rf amplitude for the optimum sensitivity is typically significantly smaller than for maximum response. The MR mode is the best experimental regime when the preamplifier noise is more significant than the effect of the SET noise, while the OS mode is the best when the preamplifier noise is negligible. Analyzing the performance dependence on the SET dc bias voltage, we have found that the best response and sensitivity are achieved at zero dc bias, though finite biasing does not change the RF-SET performance much as long as it is within the Coulomb blockade range.

We have found that the dependence of the RF-SET response on the unloaded $Q$-factor of the tank circuit has a maximum at $Q$ comparable to the simple impedance matching estimate $\sqrt{R_{\Sigma} / R_{0}}$. In contrast, the RF-SET sensitivity monotonically worsens with increase of $Q$; the dependence is approximately parabolic and the sensitivity change can reach few times (compared to the low- $Q$ case) if $Q$ is chosen too large. This means that to improve the sensitivity in an experiment, it is better to "undershoot" the $Q$-factor compared to the impedance matching case, than to "overshoot" it.

Studying the temperature dependence we have found that the RF-SET response saturates approximately at temperatures $T<0.03 e^{2} / C_{\Sigma}$, that translates into 200 $\mathrm{mK}$ for a typical capacitance value $C_{\Sigma}=300 \mathrm{aF}$. The op- 
timized sensitivity continues to improve as $T^{1 / 2}$ at lower temperatures until it reaches the quantum limitation due to cotunneling ${ }^{22,36}$ (not studied here). The "orthodox" RF-SET performance improves with decrease of the SET resistance; however, for high $Q$-factors the dependence is significantly slower than the low- $Q$ scaling $R_{\Sigma}^{-1}$ for the response and $R_{\Sigma}^{1 / 2}$ for the sensitivity.

We have analyzed the effect of the asymmetric rf biasing of the SET which leads to unequal effective capacitances of the SET and found that such asymmetry does not worsen the RF-SET performance (even slightly improves it). This answers the concern about asymmetric rf biasing raised in Ref. ${ }^{11}$.

We have analyzed the effect of the carrier frequency detuning from the resonance and found that the decrease of the RF-SET response with detuning can crudely be described by the simple linear theory (though difference in linewidth can reach 50\%); however, the estimate of the loaded $Q$-factor determining the linewidth is not simple since it significantly depends of the operation point. Even with the frequency detuning, the RF-SET performance for monitoring the reflected wave amplitude (by rectification) is found to be very similar to the case of optimal homodyne detection (one-channel mixing with the optimal phase). It is important that the mixing phase optimizing the sensitivity can be different from the phase optimizing the response. Unexpectedly, in contrast to the response decrease with detuning, the optimized sensitivity slightly improves with detuning.

We have also analyzed the operation regime for which an overtone of the incident rf wave is in resonance with the tank circuit, and found that the RF-SET performance in this regime is comparable to the performance in the conventional regime. Taking into account an advantage of the frequency separation between the incident and monitored waves, this operation mode may be experimentally useful. The theoretical proposal of the resonant overtone regime ${ }^{35}$ has been recently realized experimentally in the group of Keith Schwab. Experimental RF-SET sensitivities using the second and third resonant overtones have been found practically coinciding with the sensitivity in the conventional regime. ${ }^{39}$

Now let us compare our theoretical results for the RFSET sensitivity with the experimental value of $9 \mu e / \sqrt{\mathrm{Hz}}$ from Ref. ${ }^{12}$ for the normal-metal case (the sensitivity of the superconducting RF-SET was significantly better: $3.2 \mu \mathrm{e} / \sqrt{\mathrm{Hz}})$. Using the experimental parameters $C_{\Sigma}=267 \mathrm{aF}, R_{\Sigma}=43 \mathrm{k} \Omega$, assuming the temperature $T=70 \mathrm{mK}$, and using Fig. 6, we get the MR sensitivity of $3.2 \mu \mathrm{e} / \sqrt{\mathrm{Hz}}$ for $Q=30$ and $5.8 \mu \mathrm{e} / \sqrt{\mathrm{Hz}}$ for $Q=50$, while the OS sensitivities are $1.3 \mu e / \sqrt{\mathrm{Hz}}$ for $Q=30$ and $1.9 \mu \mathrm{e} / \sqrt{\mathrm{Hz}}$ for $Q=50$ [notice that all these numbers are significantly higher than the low- $Q$ OS estimate $0.9 \mu \mathrm{e} / \sqrt{\mathrm{Hz}}$ using Eq. (43)]. Unfortunately, it is not possible to extract the unloaded $Q$-factor value from Ref. $^{12}$; however, it seems to be between 30 and 50 . It is also not known if the RF-SET operation point was closer to the MR or OS mode (we guess the response was more important). Nevertheless, we see that the difference between the theory and experiment is few times. We guess that the difference is mainly due to the preamplifier noise. Some contribution may also come from higher effective temperature than we assumed and from a nonoptimal operation point. Another contribution to the difference may come from the neglected here effect of cotunneling, which limits the sensitivity. However, a rough estimate $^{22}$ of this limit $\delta q_{0} / \sqrt{\Delta f} \sim \sqrt{\hbar C_{\Sigma}}$ gives the value of $1 \mu e / \sqrt{\mathrm{Hz}}$, so it is unlikely to be the major reason for the difference. We hope that the further experimental progress will bring the RF-SET sensitivity really close to the theoretical limit.

There are still many theoretical questions about the RF-SET performance, not answered in this paper. For example, as seen from the above estimate, the account of cotunneling and study of the quantum operation of the RF-SET is starting to be important for experiments. The development of the theory for superconducting RF-SET is even more important since in the majority of experiments with RF-SETs the superconducting state is used. There is still no rigorous theory of the frequency dependence of the RF-SET sensitivity. It is also important to consider the backaction from the RF-SET and analyze if it can in principle be used as a quantum detector with high quantum efficiency (ideality). These problems are going to be the topics of further studies.

\section{ACKNOWLEDGMENTS}

Useful discussions with M. Blencowe, K. Likharev, and K. Schwab are gratefully acknowledged. The work was partially supported by NSA and ARDA under ARO grant DAAD19-01-1-0491 and by the Semiconductor Research Corporation grant 2000-NJ-746. The numerical calculations were partially performed on the UCR-IGPP Beowulf computer Lupin.

${ }^{1}$ D. V. Averin and K. K. Likharev, in Mesoscopic phenomena in solids, edited by B. L. Altshuler, P. A. Lee, and R. A. Webb (Elsevier, Amsterdam, 1991), p. 173.

${ }^{2}$ T. A. Fulton and G. D. Dolan, Phys. Rev. Lett. 59, 109 (1987).

${ }^{3}$ K. K. Likharev, Proc. IEEE 87, 606 (1999).

${ }^{4}$ A. N. Korotkov, Int. J. Electronics 86, 511 (1999).

${ }^{5}$ B. Starmark, T. Henning, T. Claeson, P. Delsing, and A. N. Korotkov, J. Appl. Phys. 86, 2132 (1999).

${ }^{6}$ J. Pettersson, P. Wahlgren, P. Delsing, D. B. Haviland, T. Claeson, N. Rorsman, and H. Zirath, Phys. Rev. B 53, 13272 (1996). 
${ }^{7}$ E. H. Visscher, J. Lindeman, S. M. Verbrugh, P. Hadley, J. E. Mooij, and W. van der Vleuten, Appl. Phys. Lett. 68, 2014 (1996).

${ }^{8}$ R. J. Schoelkopf, P. Wahlgren, A. A. Kozhevnikov, P. Delsing, and D. E. Prober, Science 280, 1238 (1998).

${ }^{9}$ J. Clarke, Proc. IEEE 77, 1208 (1989).

${ }^{10}$ P. Wahlgren, R J. Schoelkopf, A. A. Kozhevnikov, P. Delsing, D. E. Prober, and T. Claeson, J. Supercond. 12, 741 (1999).

11 A. Aassime, G. Johansson, G. Wendin, R. J. Schoelkopf, and P. Delsing, Phys. Rev. Lett. 86, 3376 (2001).

12 A. Aassime, D. Gunnarsson, K. Bladh, P. Delsing, and R. Schoelkopf, Appl. Phys. Lett. 79, 4031 (2001).

${ }^{13}$ A. Aassime, P. Delsing, and T. Claeson, Nanotechnology 12, 96 (2001).

${ }^{14}$ T. R. Stevenson, F. A. Pellerano, C. M. Stahle, K. Aidala, and R. J. Schoelkopf, Appl. Phys. Lett. 80, 3012 (2002).

${ }^{15}$ K. Segall, K. W. Lehnert, T. R. Stevenson, R. J. Schoelkopf, P. Wahlgren, A. Aassime, and P. Delsing, Appl. Phys. Lett. 81, 4859 (2002).

${ }^{16}$ K. Bladh, D. Gunnarsson, G. Johansson, A. Käck, G. Wendin, A. Aassime, M. Taslakov, and P. Delsing, Physica Scripta T102, 167 (2002).

${ }^{17}$ K.W. Lehnert, K. Bladh, L. F. Spietz, D. Gunnarsson, D. I. Schuster, P. Delsing, and R. J. Schoelkopf, Phys. Rev. Lett. 90, 027002 (2003).

18 T. M. Buehler, D. J. Reilly, R. P. Starret, S. Kenyon, A. R. Hamilton, A. S. Dzurak, and R. G. Clark, Microel. Eng. 67, 775 (2003).

19 T. M. Buehler, D. J. Reilly, R. P. Starret, A. D. Greentree, A. R. Hamilton, A. S. Dzurak, and R. G. Clark, condmat/0304384; cond-mat/0302085.

20 T. Fujisawa and Y. Hirayama, Appl. Phys. Lett. 77, 543 (2000).

${ }^{21}$ H. D. Cheong, T. Fujisawa, T. Hayashi, and Y. Hirayama, Appl. Phys. Lett. 81, 3257 (2002).

${ }^{22}$ A. N. Korotkov, D. V. Averin, K. K. Likharev, and S. A. Vasenko, in Single-Electron Tunneling and Mesoscopic Devices, edited by H. Koch and H. Lubbig (Springer, Berlin 1992), p. 45.

${ }^{23}$ A. N. Korotkov, Phys. Rev. B 49, 10381 (1994).

${ }^{24}$ V. A. Krupenin, D. E. Presnov, A. B. Zorin, and J. Niemeyer, J. Low Temp. Phys. 118, 287 (2000).

${ }^{25}$ L. Roschier, R. Tarkiainen, M. Ahlskog, M. Paalanen, and P. Hakonen, Appl. Phys. Lett. 78, 3295 (2001).

${ }^{26}$ P. M. Echternach, C. P. Williams, S. C. Dultz, S. Braunstein, and J. P. Dowling, Quant. Inf. Comp. 1, 143 (2001).

${ }^{27}$ A. D. Armour, M. P. Blencowe, and K. C. Schwab, Phys. Rev. Lett. 88, 148301 (2002).

${ }^{28}$ E. K. Irish and K. Schwab, cond-mat/0301252.

${ }^{29}$ R. G. Knobel and A. N. Cleland, Nature 424, 291 (2003).

${ }^{30}$ A. N. Korotkov and M. A. Paalanen, Appl. Phys. Lett. 74, 4052 (1999).

${ }^{31}$ M. P. Blencowe and M. N. Wybourne, Appl. Phys. Lett. 77, 3845 (2000).

${ }^{32}$ Y. Zhang and M. P. Blencowe, J. Appl. Phys. 91, 4249 (2002).

${ }^{33}$ Y. Zhang and M. P. Blencowe, J. Appl. Phys. 92, 7550 (2002).

34 A. B. Zorin, Phys. Rev. Lett. 86, 3388 (2001).
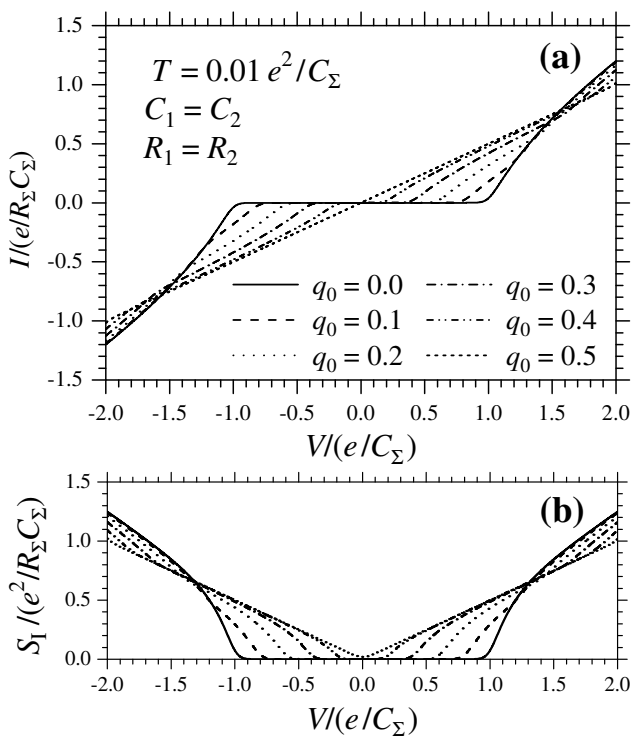

FIG. 10. (a) $I-V$ curves and (b) voltage dependence of low-frequency shot noise $S_{I}$ for the symmetric SET at $T=0.01 e^{2} / C_{\Sigma}$ for several values of the background charge $q_{0}$ from 0 to 0.5 (in units of $e$ ).

${ }^{35}$ V. O. Turin and A. N. Korotkov, cond-mat/0305012; to be published in Appl. Phys. Lett.

${ }^{36}$ M. H. Devoret and R. J. Schoelkopf, Nature 406, 1039 (2000).

37 A. N. Korotkov, R. H. Chen, and K. K. Likharev, J. Appl. Phys. 78, 2520 (1995).

${ }^{38}$ R. Knobel, C. S. Yung, and A. N. Cleland, Appl. Phys. Lett. 81, 532 (2002).

${ }^{39}$ K. Schwab, private communication.

\section{APPENDIX: CURRENT AND NOISE CALCULATIONS FOR THE SET}

The SET current $I$ and its low frequency shot noise $S_{I}$ have been calculated using the orthodox theory of singleelectron tunneling, ${ }^{1}$ assuming that a stationary state of the SET is achieved at any moment of time (i.e. $I / e \ll$ $\omega)$. We assume normal metal (non-superconducting) case and calculate the tunneling rates $\Gamma_{1,2}^{ \pm}(m)$ of electron tunneling to $(+)$ or from $(-)$ the island through the first or second junction as ${ }^{1}$

$$
\begin{aligned}
\Gamma_{j}^{ \pm}(m) & =\frac{W_{j}^{ \pm}(m)}{e^{2} R_{j}\left[1-\exp \left(-W_{j}^{ \pm}(m) / T\right)\right]}, \\
W_{j}^{ \pm}(m) & =\frac{e^{2}}{C_{\Sigma}}\left[\mp(-1)^{j} \frac{V_{b} C_{1} C_{2}}{e C_{j}}-\frac{1}{2} \mp m \mp \frac{q_{0}}{e}\right],
\end{aligned}
$$

where $m$ is the number of extra electrons on the SET, $j=1,2$ denotes the junction, and $T$ is the temperature.

The current [Fig. 10(a)] is calculated as 


$$
I=e \sum_{m}\left[\Gamma_{1}^{+}(m)-\Gamma_{1}^{-}(m)\right] \sigma_{s t}(m),
$$

where the stationary probability distribution of the charge states $\sigma_{s t}(m)$ satisfies equations

$$
\sigma_{s t}(m) \sum_{j} \Gamma_{j}^{+}(m)=\sigma_{s t}(m+1) \sum_{j} \Gamma_{j}^{-}(m+1)
$$

and $\sum_{m} \sigma_{s t}(m)=1$.

The low-frequency spectral density $S_{I}$ of the SET current [Fig. 10(b)] can be calculated as ${ }^{23}$

$$
\begin{aligned}
& S_{I}=-4 e^{2} \sum_{m, m^{\prime}}\left[\Gamma_{1}^{+}\left(m^{\prime}\right)-\Gamma_{1}^{-}\left(m^{\prime}\right)-I / e\right]\left(\hat{\boldsymbol{\Gamma}}^{-1}\right)_{m^{\prime} m} \\
& \quad \times\left[\Gamma_{1}^{+}(m-1) \sigma_{s t}(m-1)-\Gamma_{1}^{-}(m+1) \sigma_{s t}(m+1)\right. \\
& \left.-(I / e) \sigma_{s t}(m)\right]+2 e^{2} \sum_{m} \sigma_{s t}(m)\left[\Gamma_{1}^{+}(m)+\Gamma_{1}^{-}(m)\right],
\end{aligned}
$$

where $\hat{\boldsymbol{\Gamma}}$ is the three-diagonal matrix of the SET charge evolution, $\boldsymbol{\Gamma}_{k m}=\sum_{j}\left[\delta_{m, k-1} \Gamma_{j}^{+}(m)+\delta_{m, k+1} \Gamma_{j}^{-}(m)-\right.$ $\left.\delta_{m, k}\left[\Gamma_{j}^{+}(m)+\Gamma_{j}^{-}(m)\right]\right]$. Notice that Eq. (A5) can be easily used numerically even though the matrix $\hat{\Gamma}$ is singular and therefore does not have a unique inverse; Eq. (A5) is constructed in a way that the non-uniqueness is not important, and therefore the standard algorithm for solving a linear system of equations with three-diagonal matrix can be readily used. (There is no problem of the matrix singularity at a finite frequency $\omega_{s}$, when the inverse of the matrix $\hat{\boldsymbol{\Gamma}}-i \omega_{s} \hat{\mathbf{1}}$ should be calculated.) Actually, instead of using Eq. (A5), we have used a somewhat faster algorithm for calculation of $S_{I}$, described in Section VII of Ref. ${ }^{23}$. 